\title{
Failure Analysis of the Escalator Driving Chain in an Escalator Reversal Accident
}

\author{
Bo $\mathrm{Li}^{1, \mathrm{a}}$, Xiao Liang ${ }^{1, \mathrm{~b}}$ \\ ${ }^{1}$ Shanghai Institute of Special Equipment Inspection and Technical Research, 399 North Nujiang \\ Road, Shanghai 200333, PR China \\ alibo@ssei.cn, bliangxiao@ssei.cn
}

Keywords: Failure analysis; Escalator; Chain; decarburization; Fatigue crack

\begin{abstract}
An escalator accident occurred in a subway station of Shanghai City, resulting into that the running direction of the escalator sudden reversals, resulting in that some passengers were injured. In this paper, the failure analysis of chain plate of broken chain drive chain is analyzed. The escalator driving chain breakage is caused by the material deterioration of the transition chain link plate. The accident escalator driving chain adopts the transition segment, so that the stress concentration effects are exerted at the position of the transition linked chain plate bending corner. The decarburization phenomenon exists within the chain plate surface, resulting into that the chain plate generates fatigue cracking in the service process. The depth of the decarburization surface layer was measured as about $90 \mu \mathrm{m}$. The surface-layer material softening accelerated the occurrence of the fatigue fracture.
\end{abstract}

\section{Introduction}

The escalator reversal accident refers to a phenomenon of that the escalator conducts the operation of non man-made changes against its normal motion direction. The escalators which either at upward or downward, are likely to be reversed. However, the escalators at the upstream conditions have higher probability for reversal accidents. The reversal accident has a high risk that induces the falling, rolling, extrusion, or stampede of the escalator passengers.

In the present paper, an accident failure analysis of an escalator in a subway station is completed. The accident escalator ran in the process of sudden change into a reversed direction to the downward, resulting in that number of passengers standing on the escalator step fell in and were injured.

According to the accident scene exploration and investigation, it was found that the escalator drive chain, which was between the actuating device and the step chain sprocket, was fractured. Therefore, the failure cause should be revealed.

\section{Failure Analysis of the Driven Chain}

Macro-structural Examination. The fractured escalator drive chain belongs to the chain model of double row roller chain (Specification No.20A-2). The chain pitch is $31.75 \mathrm{~mm}$, with a long chain pin width of about $83.2 \mathrm{~mm}$. The breakage of the double drive chain occurred during its service process. The fracture position located at the transition section of the double row roller chain(Fig.1, and Fig.2). Moreover, the chain pin next to the transition chain plate was plastic deformed at severe locations where suffered partially forces from the drive chain of the escalator.

Fracture Surface Observation. The fracture surface morphology of the four fractured transition chain plates were observed in detail, as shown in Fig.3. The four fracture surface were all silver-grey in color. The crack patterns on the fracture surfaces extended along the cracking direction by the bending angle. The crack extensions were all initially from the chain plate surfaces into the inwards, as indicated by the red arrows in Fig.3. The SEI (Secondary Electron Imaging) of the fracture surfaces claimed that the typed fatigue striations were formed and presented on them, as shown in Fig.3. The parallel distribution of fatigue striations on the quasi-cleavage fracture surfaces implied the fatigue cracks were initiated and propagated before that the whole driving chain failure and the final accident occurred on the escalator. 


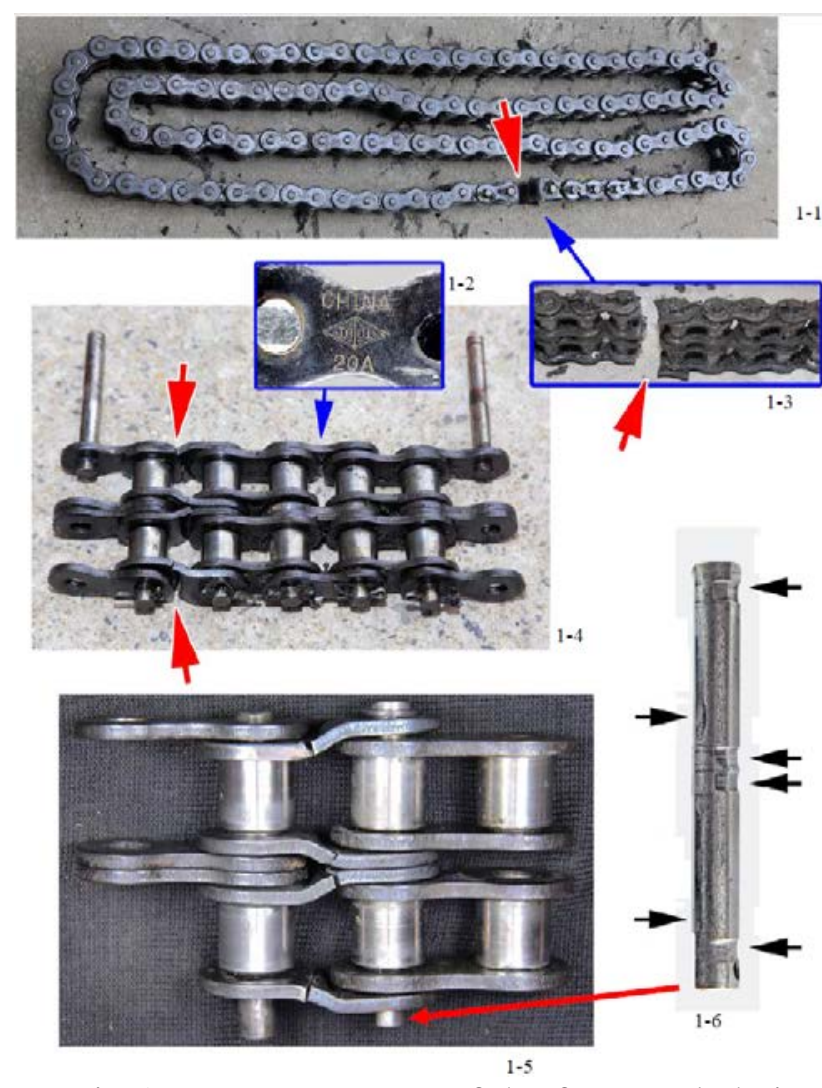

Fig.1 Macro-structure of the fractured chain

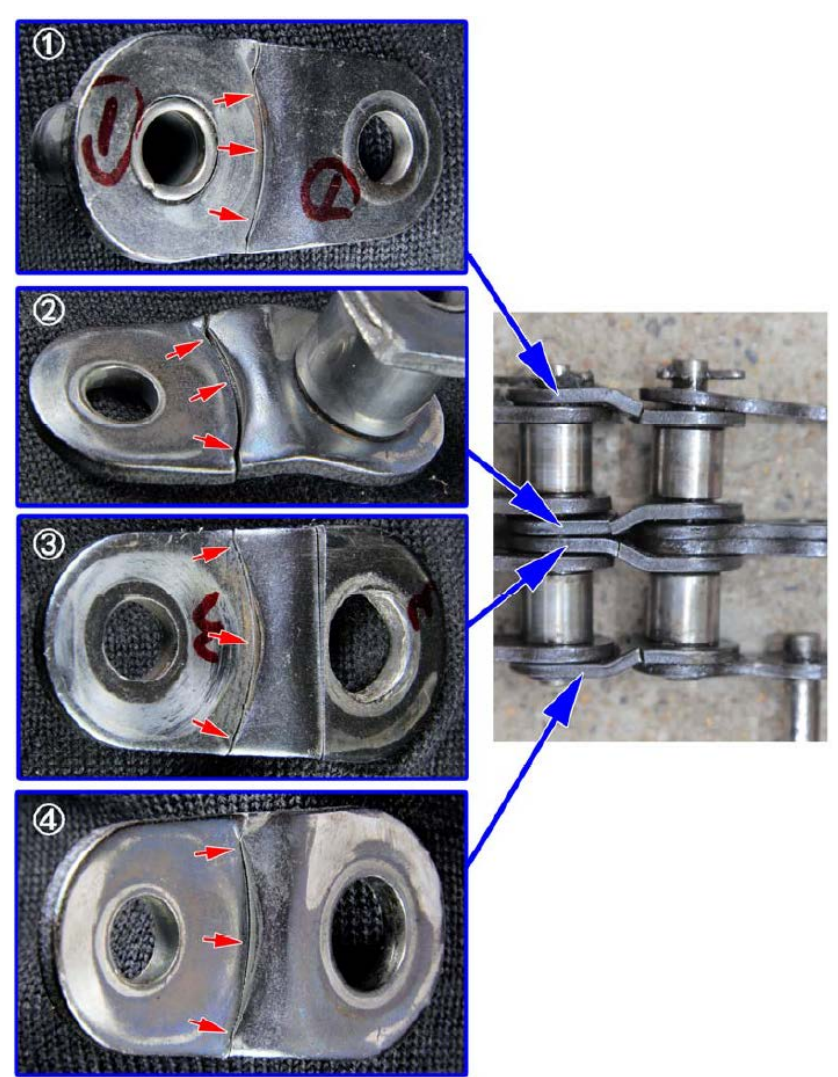

Fig.2 The fractured transition chain plates

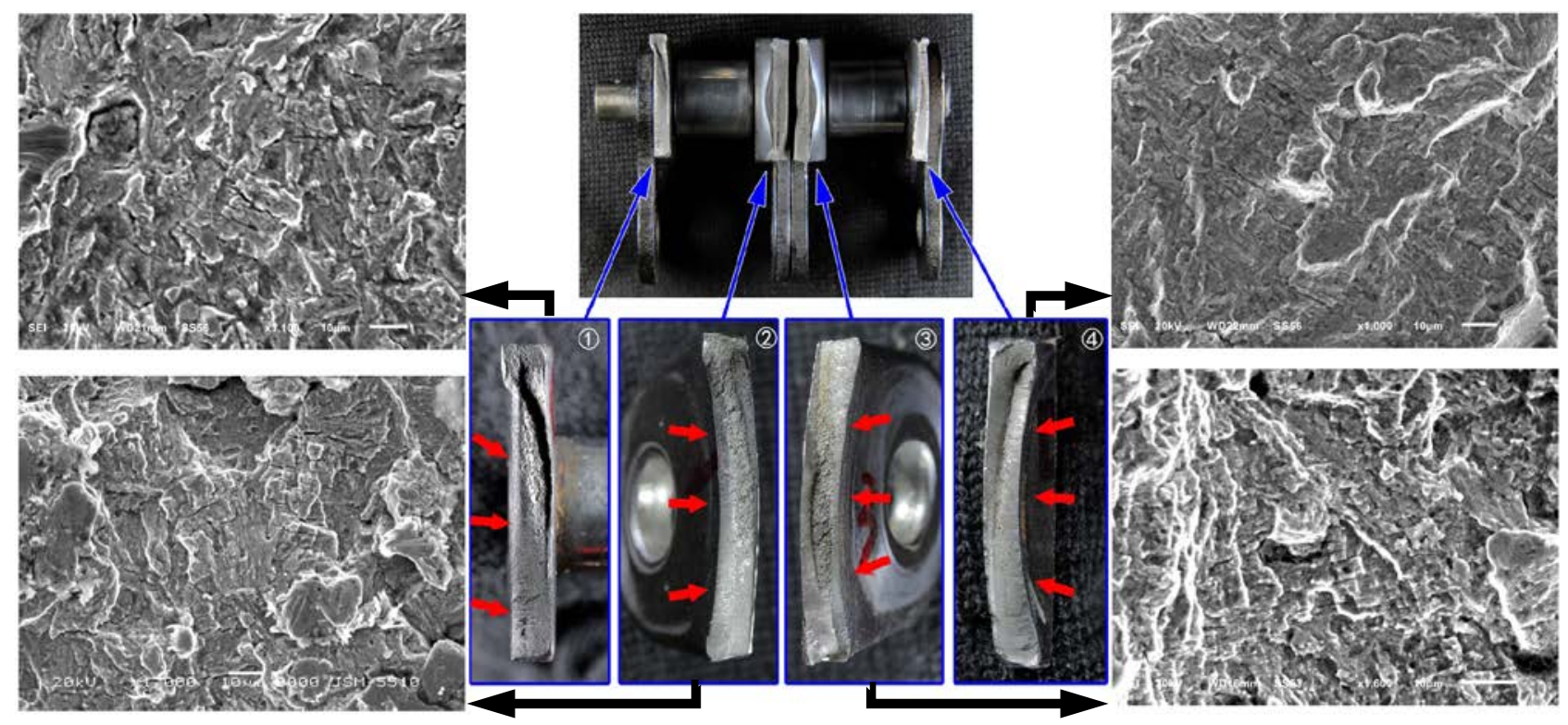

Fig.3 Fracture surface morphology of the fractured chain plates

Micro-structural Analysis. The section micro-structure of the transitional chain plate, closing to the fracture position, was analyzed in detail and shown in Fig.4. The cross-section morphology of corresponding sample (the No.(4) chain plate in Fig.2 and Fig.3) indicated that the decarburization phenomenon was existed on the outer surface. The micro-structure of the chain plate was Ferrite with a small amount of Sorbite. The micro cracking originated from the decarburization surface layer was given as Fig.4c. The depth of the decarburization surface layer was measured as about $90 \mu \mathrm{m}$. And the micro cracks also existed within the decarburization surface layer of the bending area on the other side of the chain plate, as shown in Fig.4e and Fig.4f. 


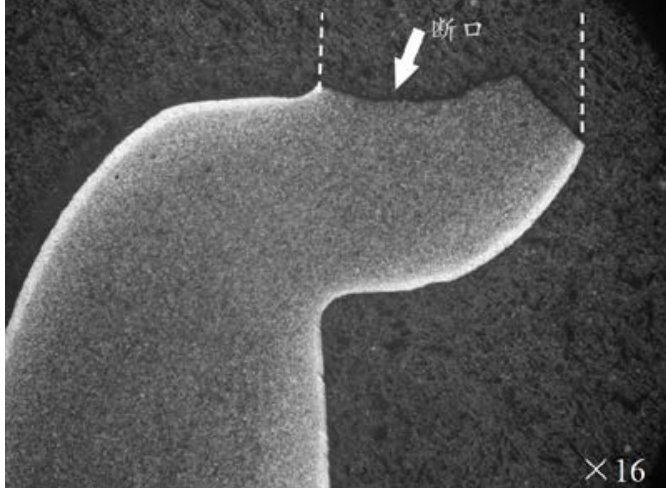

(a)

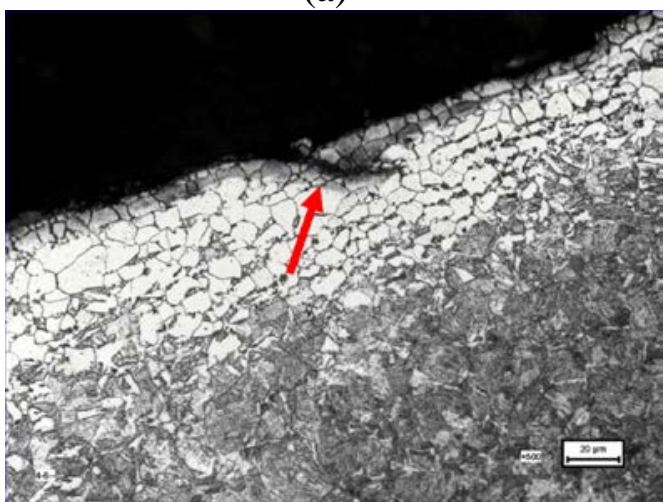

(c)

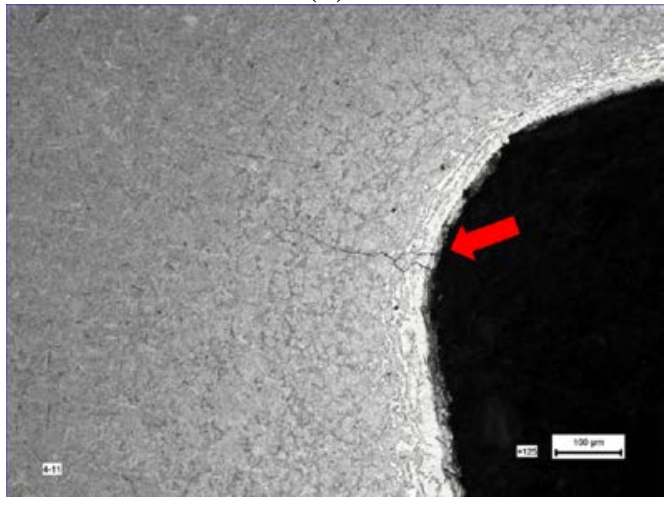

(e)

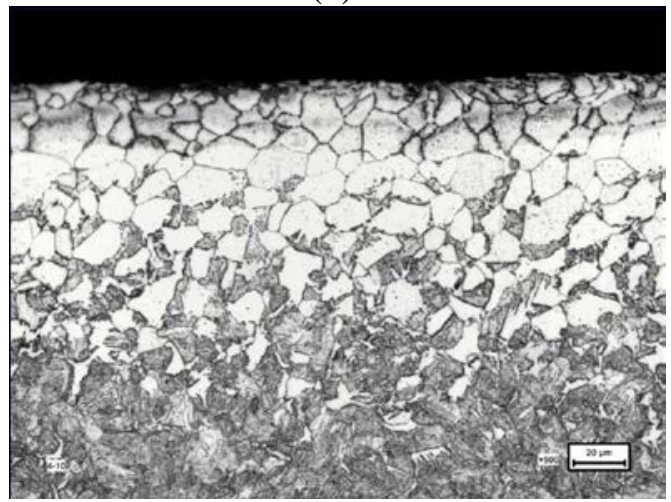

(g)

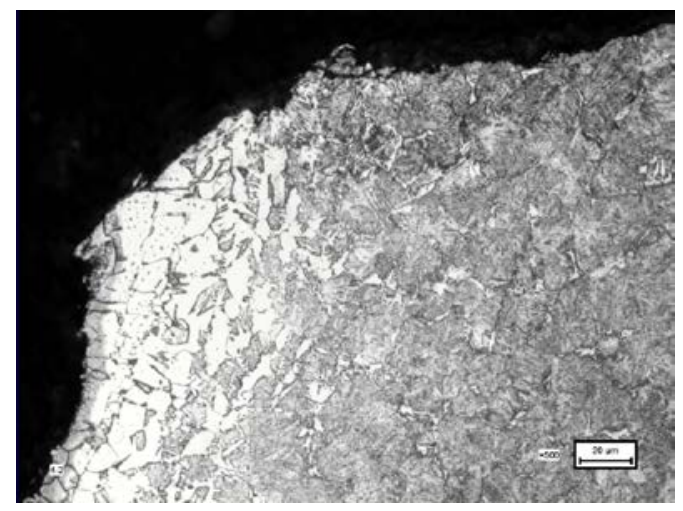

(b)

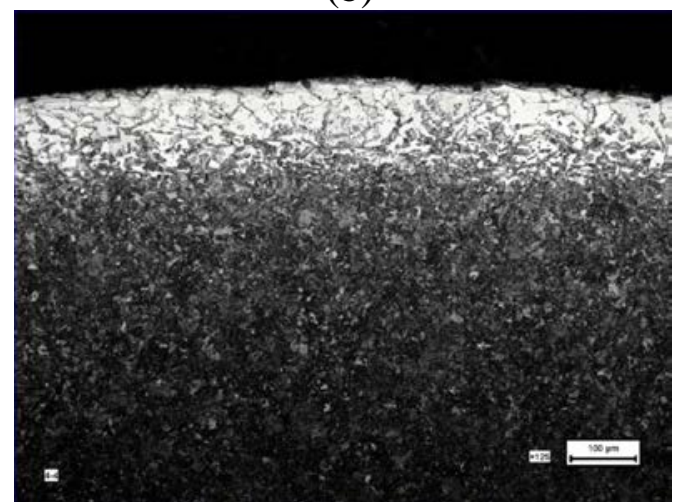

(d)

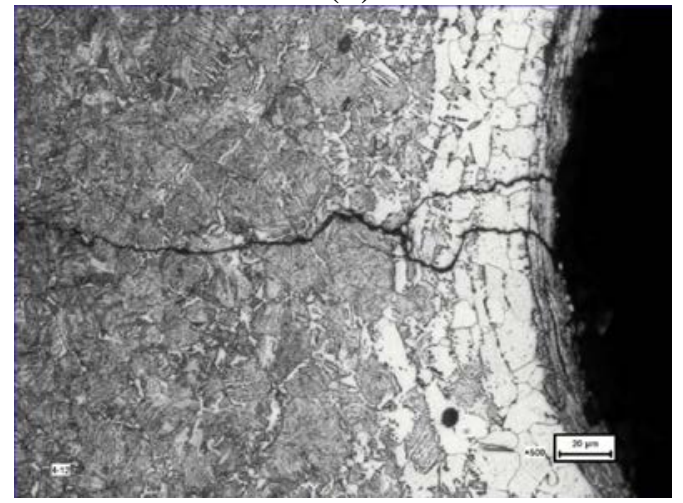

(f)

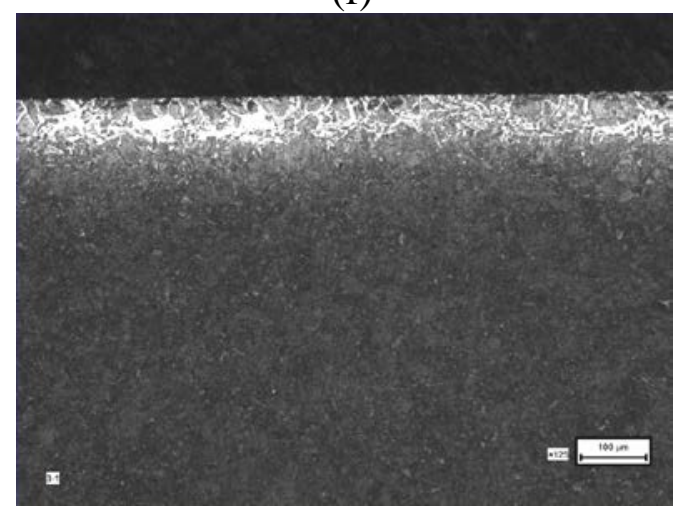

(h)

Fig.4 The micro-structures of the failed transitional chain plate with the decarburization surface layer and micro cracks.

Hardness Measurement. The results of Vickers hardness measurements on the cross section of the related samples are shown in Table 1 . The Vickers hardness values of the surface layer of the samples are generally higher than those of the inner part of the samples. It indicates that the decarburization surface layer induces the material softening of the chain plate surface. 
Table 1 The Vickers hardness values of the measured samples

\begin{tabular}{|c|c|c|}
\hline Sample & $\begin{array}{l}\text { The Vickers hardness values of the } \\
\text { inner part of the samples }\end{array}$ & $\begin{array}{l}\text { The Vickers hardness values of the } \\
\text { surface layer of the samples }\end{array}$ \\
\hline The failed chain plate of No.(1) & $430 ; 423 ; 430$ & $382 ; 370 ; 369$ \\
\hline The failed chain plate of No.(2) & $410 ; 419 ; 411$ & 377; 391; 388 \\
\hline The failed chain plate of No.(3) & $425 ; 425 ; 413$ & $374 ; 382 ; 379$ \\
\hline The failed chain plate of No.(4) & $426 ; 424 ; 424$ & $162 ; 211 ; 191$ \\
\hline $\begin{array}{l}\text { The chain plate without failure } \\
\text { but with the decarburization }\end{array}$ & $424 ; 434 ; 420$ & $350 ; 339 ; 412$ \\
\hline
\end{tabular}

\section{Failure Analysis Results and Discussion}

According to the metallographic and micro-structure scanning electron microscope analysis, it is claimed that no abnormal inclusion exists within the driving chain plates. Therefore, the micro inclusion is not the main factor inducing the fracture of the chain. The metallographic observation and hardness measurement results showed that the drive chain plate has the varying degree of surface decarburization defects. The surface decarburized layer can be inferred that the anti-fatigue ability of the chain plates is deviated. The cause of decarbonization phenomenon is directly related to the unreasonable heat treatment process of the chain. Decarburization (or decarbonization) is the process opposite to carburization, namely the reduction of carbon content. The term is typically used in metallurgy, describing the reduction of the carbon content in metals (usually steel). Decarburization is an important process during the heat treatment of steels. It can be divided into three separated events: dissolution of carbides, diffusion of carbon through the iron matrix, and surface reactions. Decarburization occurs when the metal is heated to temperatures of $700{ }^{\circ} \mathrm{C}$ or above when carbon in the metal reacts with gases containing oxygen or hydrogen. Decarburization can be either advantageous or detrimental, depending on the application for which the metal will be used. It is thus both something that can be done intentionally as a step in a manufacturing process, or something that happens as a side effect of a process (such as rolling) and must be either prevented or later reversed (such as via a carburization step). The decarburization mechanism can be described as three distinct events: the reaction at the steel surface, the interstitial diffusion of carbon atoms and the dissolution of carbides within the steel. The transition chain of the sample drive chain is fatigue fracture. The fatigue fracture of the multiple sources starting with the stress concentrations. Therefore, it is concluded that the surface decarburization will reduce the surface fatigue strength of chain plate, on the basis of the observation of the fracture surface analysis. At the same time, it is found that the cracking phenomenon can be inferred from the corner of the transition chain plate. The bending angle of the transition chain plate has obvious stress concentration effect in the operation.

\section{References}

[1] H.D. Alvarenga, T.V.D. Putte and N.V. Steenberge: Metallurgical \& Materials Transactions A Vol.46A(2015), p. 123.

[2] R.W. Klopp: Failure Analysis of Redundant Escalator Chain Pin Retention Mechanisms, ASME 2011 International Mechanical Engineering Congress and Exposition. 2011, p. 407.

[3] T.G. Kim, S.B. Lee, H.C. Lee: Safety \& Health at Work Vol.1(2010), p. 43.

[4] C. Franco, A. Rita, C. Mauro: IEEE Computer Society(2011), p. 1221.

[4] X.J. Hao, W. Yin, M. Strangwood: Scripta Materialia Vol.58(2008), p. 1033. 